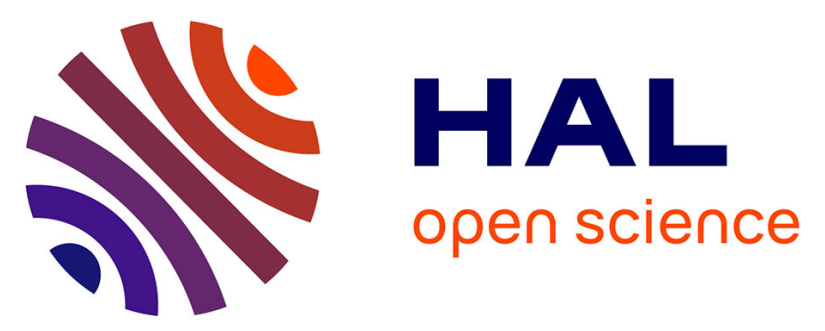

\title{
Measurement of dispersion curves of circumferential guided waves radiating from curved shells: Theory and numerical validation
}

Mathieu Chekroun, Jean-Gabriel Minonzio, Claire Prada, Pascal Laugier, Quentin Grimal

\section{To cite this version:}

Mathieu Chekroun, Jean-Gabriel Minonzio, Claire Prada, Pascal Laugier, Quentin Grimal. Measurement of dispersion curves of circumferential guided waves radiating from curved shells: Theory and numerical validation. Journal of the Acoustical Society of America, 2016, 139 (2), pp.790. 10.1121/1.4941652 . hal-01305025

\section{HAL Id: hal-01305025 \\ https://hal.sorbonne-universite.fr/hal-01305025}

Submitted on 20 Apr 2016

HAL is a multi-disciplinary open access archive for the deposit and dissemination of scientific research documents, whether they are published or not. The documents may come from teaching and research institutions in France or abroad, or from public or private research centers.
L'archive ouverte pluridisciplinaire HAL, est destinée au dépôt et à la diffusion de documents scientifiques de niveau recherche, publiés ou non, émanant des établissements d'enseignement et de recherche français ou étrangers, des laboratoires publics ou privés. 


\section{Measurement of dispersion curves of circumferential guided waves radiating from curved shells: Theory and numerical validation}

\section{Running Title : Circumferential guided waves on curved shells}

March 23, 2015

\section{Mathieu Chekroun ${ }^{1}$}

Laboratoire d'Acoustique de l'Université du Maine UMR-CNRS 6613, av. Olivier Messiaen, 72085 Le Mans cedex 9, France

\section{Jean-Gabriel Minonzio}

Sorbonne Universités, UPMC Univ Paris 06, CNRS UMR 7371, INSERM UMR_S 1146, Laboratoire d'Imagerie Biomédicale, F-75005, Paris, France

\section{Claire Prada}

Institut Langevin, Ecole Superieure de Physique et de Chimie Industrielles Paris Tech, Universite Denis Diderot Paris 7, CNRS 7587, 1 rue Jussieu, F-75005 Paris, France

\section{Pascal Laugier and Quentin Grimal \\ Sorbonne Universités, UPMC Univ Paris 06, CNRS UMR 7371, INSERM UMR_S 1146, Laboratoire d'Imagerie Biomédicale, F-75005, Paris, France}

\footnotetext{
${ }^{1}$ Author to whom correspondence should be addressed. Electronic mail: mathieu.chekroun@univlemans.fr
} 


\begin{abstract}
A method is proposed to evaluate in a non-contact way the phase velocity dispersion curves of circumferential waves around a shell of arbitrary shape immersed in a fluid. No assumptions are made about the thickness nor the material of the shell. A geometrical model is derived to describe the shape of the radiated wavefronts in the surrounding fluid, and predict the positions of its centers of curvature. Then the time-reversal principle is applied to recover these positions and to calculate the phase velocity of the circumferential waves. Numerical finite-difference simulations are preformed to evaluate the method on a circular and on an elliptic thin shells. The different dispersion curves branches can be recovered with an error of less than $10 \%$.
\end{abstract}

PACS numbers : 43.20.Mv, 43.30.Jx, 43.40.Rj 


\section{I INTRODUCTION}

2

Circumferential waves are interface-type waves propagating around a cylindrical object. They can be classified into two groups : internal and external waves. The external waves (generalized Stoneley waves, Franz waves, creeping waves) reside essentially at the external interface of the object. Their velocity is close to that of the waves in the surrounding medium (water in the present case). The internal waves are analogous to Rayleigh waves in the case of a plain cylinder or to Lamb waves in the case of shells. They depend on the material constituting the scatterer, and are thus suitable to perform evaluation or characterization of the material.

Circumferential waves are generated when an incident wave hits the interface at a critical angle of incidence $\beta$ where, following Snell's law, $\beta=\operatorname{asin} \frac{c_{0}}{c_{\phi}}, c_{0}$ and $c_{\phi}$ denoting the phase velocities of acoustic waves in the surrounding medium and of the circumferential wave, respectively. During the propagation, circumferential waves radiate in the surrounding medium at the same angle $\beta$, and thus contribute to the scattered field when a revolution object is hit by an incident wave. This can be underlined for example by applying a Sommerfeld-Watson transform on the classical partial wave decomposition of the scattered field : the contributions may be separated into geometrical components (specular reflection, transmission) and circumferential waves $[1,2,3]$. At high frequencies, the contribution of the radiated circumferential waves in a given point may be evaluated using the Geometrical Theory of Diffraction [4], which has especially been applied on spheres [5].

Visualization of the radiated waves can be done using the Schlieren method, allowing the observation of the wavefronts shape $[6,7,8]$. The geometrical shape of these wavefronts have been studied in $[6,9,10]$. In particular, in the case of a circular cylinder of radius $a$, the wave front equation have been shown to be an involute of a circle of radius $(a \sin \beta)$ with the same center as the cylinder [11]. Figure 1 (adapted from [6]) shows the geometrical construction of the radiated wavefronts.

The aim of this paper is to describe a method to evaluate the dispersion curves of circumferential guided waves in thin shells of arbitrary geometry immersed in a fluid, observed at a distance by an array of receivers. The method was initially suggested by Thomas et 




Figure 1: Geometrical construction of the radiated wavefront by a circumferential wave around a circular cylinder (adapted from ref. [6]).

al. [12] for thin circular cylindrical shells, based on the time reversal of the waves radiated in the fluid by the circumferential waves. Building on the later, Prada al. [13] were able to measure several lamb modes dispersion branch by applying the DORT method (a French acronym for Décomposition de l'Opérateur de Retournement Temporel), taking advantages of the analysis of the iterative time reversal operators [14]. In particular, the DORT method allowing the separation of the different contributions in the case of multi-modal propagation of the circumferential waves. Experimental application was successfully conducted on a thin cylindrical steel shell $[12,13]$ and on a thicker cylindrical bone mimicking phantom $[15,16]$. The later work on a bone mimicking phantom was motivated by the potential to measure the properties of the cortical shell of the femoral neck by an ultrasound scanner, in the context of osteoporosis fracture risk assessment. In order to accommodate the complex geometry of the femoral neck, the method of measurement of circumferential waves was first extended from circular shell to a particular case of elliptic shells where the major axis of the ellipse is parallel to the transducer array [17]. In the present paper, the basis of the method is revisited and a generalization is proposed to evaluate cylindrical objects, the external cross section of which can be circular as in $[12,13,15]$, elliptic, or of arbitrary geometry. 


\section{CONFIGURATIONS}

The method introduced in this article processes the wavefront radiated in an acoustic medium by a circumferential wave to recover its phase velocity. It can in principle be applied to any cylindrical shell of arbitrary cross section. For the purpose of illustration the details of the configurations given below will be used throughout the remaining of the article.

Three different cylindrical shells will be considered

- A circular shell of radius $a=10 \mathrm{~mm}$;

- An elliptical shell of major semi-axis $r_{1}=10 \mathrm{~mm}$ and minor semi-axis $r_{2}=7.5 \mathrm{~mm}$, with the major axis oriented parallel to the array $\left(\alpha=0^{\circ}\right)$;

- The same elliptical shell, but with its major axis oriented at an angle $\alpha=30^{\circ}$.

In the case of the ellipses, $\alpha$ denotes the angle between the major axis and an axis parallel to the array. For all the cases, the thickness of the shell is constant and taken to $e=1 \mathrm{~mm}$. With the typical mean radius of the shell $a=10 \mathrm{~mm}$, the ratio $e / a$ is small (weak curvature of the shell). As a consequence, the properties of the circumferential guided modes will be close to those of the leaky Lamb modes in a plane plate $[18,19,20]$. With the frequency range of interest centered around $f_{0}=1 \mathrm{MHz}$ ( $k e \simeq 4$ in water), three Lamb modes will be observed. The external fluid surrounding the shell is water, and the shell is considered as filled with air. The chosen properties of the medium constituting the shell are close to those of the cortical bone [21] (table 1).

Table 1: Material properties used for simulations (Longitudinal velocity $c_{L}$, shear velocity $c_{T}$ and density $\rho$ )

\begin{tabular}{lccc}
\hline & $c_{L}$ & $c_{T}$ & $\rho$ \\
& $\left(\mathrm{m} \cdot \mathrm{s}^{-1}\right)$ & $\left(\mathrm{m} . \mathrm{s}^{-1}\right)$ & $\left(\mathrm{g} . \mathrm{cm}^{-3}\right)$ \\
\hline \hline Water & 1500 & - & 1 \\
Shell & 4000 & 1800 & 1,85 \\
\hline
\end{tabular}

A 128 elements transducer array of total width $D=64 \mathrm{~mm}$ is used to record the radiated wavefronts in order to determine the phase velocity $c_{\varphi}$ by applying the time reversal principle. 
This transducer is located at a distance $F=60 \mathrm{~mm}$ from the center of the object. The general configuration for the simulations is presented in fig. 2 .

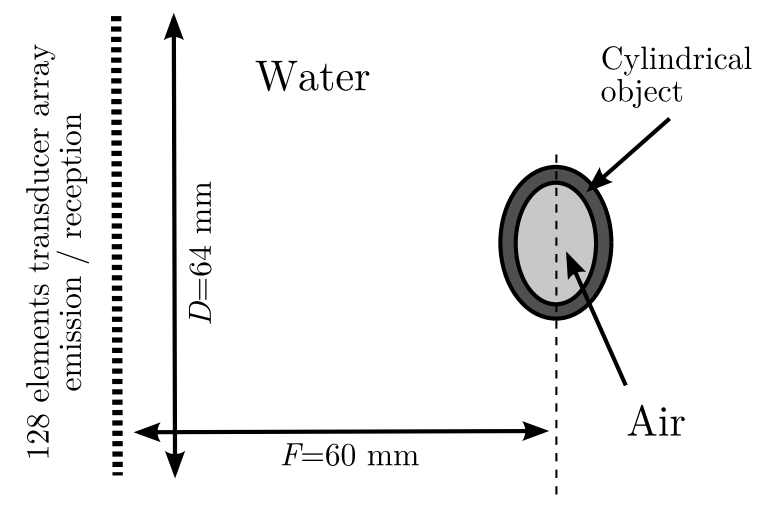

Figure 2: General configuration of the setup used to evaluate the dispersion curves of the circumferential guided waves around a thin cylindrical shell.

68

\section{CASE OF CIRCUMFERENTIAL WAVES IN ELLIPTICAL SHELLS OB- SERVED AT A VERY LARGE DISTANCE}

At a distance of the shell, the radiated rays seem to arise from a virtual point source inside the object [22]. The position of this virtual source depends on both the phase velocity of the circumferential wave (through the critical angle $\beta$ ) and the geometrical shape of the shell. By applying the time reversal principle [23] it is possible to retrieve the position of this virtual source as a focal spot. The position of the latter is then used to recover the phase velocity $c_{\varphi}$ for each frequency [12]. In the case of a circular shell, the incident plane wave generates a pair of symmetrical circumferential waves traveling in opposite directions (clockwise and counterclockwise), providing two distinct virtual sources (see fig. 3). If the assumption is made that the receivers are located toward the infinity in the left, the distance $d$ between these virtual sources is linked with the phase velocity $c_{\varphi}$ with the relation

$$
c_{\varphi}=\frac{2 a c_{0}}{d}
$$

$c_{0}$ denoting the celerity in the surrounding fluid and $a$ the external radius of the cylinder.

The same argument as in [12] has been applied to the case of an elliptic shell with major semi-axis $r_{1}$ and minor semi-axis $r_{2}$, and which major axis is parallel to the transducer 


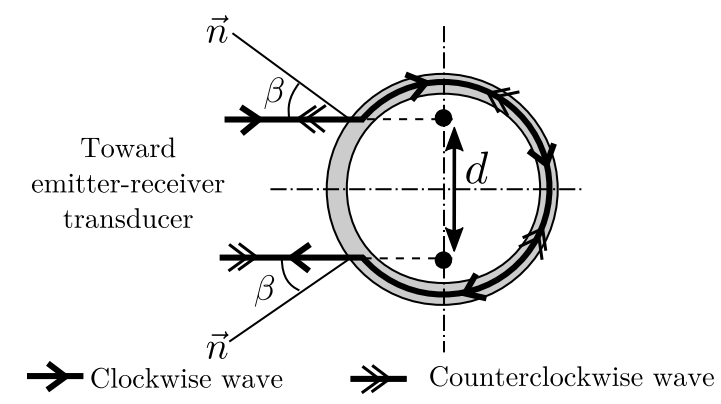

Figure 3: Principle of the generation and radiation of circumferential waves around a thin circular cylinder.

84

array [17]. This leads to the explicit relation (2)

$$
c_{\varphi}=\frac{c_{0}}{r_{2} d} \sqrt{4 r_{1}^{4}+\left(r_{2}^{2}-r_{1}^{2}\right) d^{2}},
$$

linking the dimensions of the ellipse $r_{1}, r_{2}$, the celerity $c_{0}$ in the surrounding fluid, and the distance $d$ between the two focal spots obtained by time reversal. In particular, the result of eq.(1) for the circular shell is retrieved when putting $r_{1}=r_{2}=a$ in eq (2).

In the case of an elliptic shell with an arbitrary orientation compared to the array, or in the case of a shell of arbitrary cross section, the derivation of a relation such as eq. (2) is much more complex. In particular, the symmetry with respect to the horizontal axis is lost, which imposes the upper and lower parts of the shell to be considered separately. Moreover in equations (1) or (2), the assumption is made that the transducer array is located at an infinite distance, so that the radiation of the circumferential wave is considered to be parallel to the horizontal axis, which is obviously not the case, in particular for the receivers located at the edges of the array. These limitations are addressed in the rest of the paper.

\section{GEOMETRICAL MODEL OF THE RADIATED WAVEFRONT}

In this section we develop a model to describe the radiation of circumferential guided waves using geometrical acoustics. 


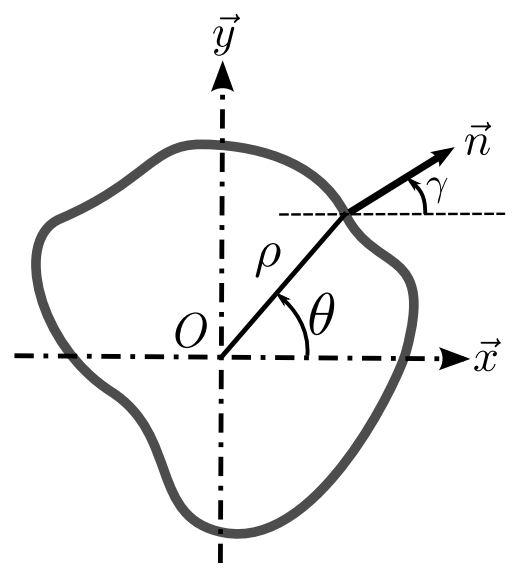

Figure 4: Notations used to describe the radiation of a circumferential wave around a shell of arbitrary cross section.

\section{A Description of the shell}

We consider a thin cylindrical shell of arbitrary cross section, the edge of which is described by the polar curve $\rho(\theta)$ (see fig. 4). It corresponds to the Cartesian coordinates

$$
\left\{\begin{array}{l}
x(\theta)=\rho(\theta) \cos \theta \\
y(\theta)=\rho(\theta) \sin \theta
\end{array}\right.
$$

The external normal vector $\vec{n}$ for each point of the shell is defined by the relation

$$
\vec{n}(\theta)=\left\{\begin{array}{l}
n_{x}=\frac{y^{\prime}}{\sqrt{y^{\prime 2}+x^{\prime 2}}}=\frac{\rho^{\prime} \sin \theta+\rho \cos \theta}{\sqrt{\rho^{\prime 2}+\rho^{2}}} \\
n_{y}=\frac{-x^{\prime}}{\sqrt{y^{\prime 2}+x^{\prime 2}}}=\frac{\rho \sin \theta-\rho^{\prime} \cos \theta}{\sqrt{\rho^{\prime 2}+\rho^{2}}}
\end{array}\right.
$$

where the ' denotes the derivative with respect to $\theta \cdot \vec{n}$ can also be defined by its orientation $\gamma$ relative to the horizontal axis $\vec{x}$ (see Fig. 5), expressed as

$$
\tan \gamma(\theta)=\frac{n_{y}}{n_{x}}=\frac{\rho \sin \theta-\rho^{\prime} \cos \theta}{\rho^{\prime} \sin \theta+\rho \cos \theta} .
$$

Finally, the arc length $s\left(\theta_{1}, \theta_{2}\right)$ between two points of the shell defined by the polar angles $\theta_{1}$ and $\theta_{2}$ is

$$
s\left(\theta_{1}, \theta_{2}\right)=\int_{\theta_{1}}^{\theta_{2}} \sqrt{\rho^{\prime}(\theta)^{2}+\rho(\theta)^{2}} d \theta .
$$




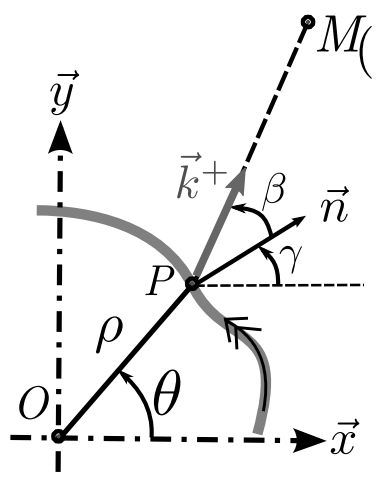

Counterclockwise

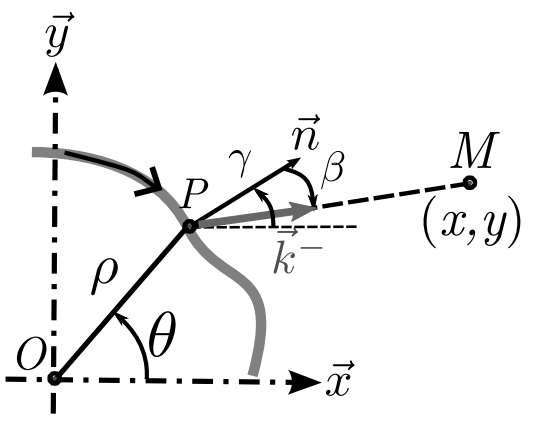

Clockwise

Figure 5: Direction of the radiation $\vec{k}^{+}$and $\vec{k}^{-}$for a circumferential wave propagating counterclockwise and clockwise.

\section{B Wavefront equation}

We consider a circumferential guided wave propagating at the phase velocity $c_{\varphi}$ and frequency $\omega$ around the shell. This guided wave radiates in the surrounding medium at the angle $\beta$. The radiated wave has the velocity $c_{0}$ in the surrounding medium. As the circumferential waves may propagate in both clockwise (denoted as superscript + ) and counterclockwise (denoted as -) directions along the shell, radiation in the directions $\vec{k}^{-}$and $\vec{k}^{+}$will be considered (fig. 5).

The time $t=0$ is taken when the circumferential wave is at $\theta=0$ in the shell. At the time $t$, a given point $M$ of coordinates $(x, y)$ is reached by a wave radiated in straight line from the point $P$ of coordinates $(\rho, \theta)$ on the shell (Fig. 5). The circumferential wave has traveled along a distance $s(0, \theta)$ at the celerity $c_{\varphi}$ in the shell and the radiated wave has traveled from $P$ to $M$ at the celerity $c_{0}$.

The radiated wavefronts are the set of points $M$ reached at the time $t$. Considering polar angles $\theta$ in $[0 ; 2 \pi]$, the coordinates of the radiated wavefront $\mathbf{F}^{+}(t, \theta)$ for a counterclockwise circumferential wave is (eq. (7)):

$$
\mathbf{F}^{+}(t, \theta)\left\{\begin{array}{l}
x(t, \theta)=\rho(\theta) \cos \theta+c_{0}\left(t-\frac{s(0, \theta)}{c_{\varphi}}\right) \cos (\gamma(\theta)+\beta) \\
y(t, \theta)=\rho(\theta) \sin \theta+c_{0}\left(t-\frac{s(0, \theta)}{c_{\varphi}}\right) \sin (\gamma(\theta)+\beta)
\end{array}\right.
$$


and a similar result for $\mathbf{F}^{-}(t, \theta)$ radiated by a clockwise circumferential wave (eq. (8)):

$$
\mathbf{F}^{-}(t, \theta)\left\{\begin{array}{l}
x(t, \theta)=\rho(\theta) \cos \theta+c_{0}\left(t-\frac{s(\theta-2 \pi, 0)}{c_{\varphi}}\right) \cos (\gamma(\theta)-\beta) \\
y(t, \theta)=\rho(\theta) \sin \theta+c_{0}\left(t-\frac{s(\theta-2 \pi, 0)}{c_{\varphi}}\right) \sin (\gamma(\theta)-\beta)
\end{array}\right.
$$

In the two formulations (7) and (8), only the physically relevant solutions are considered to respect causality. These corresponds to the terms describing the propagation in water from $P$ to $M$, where the quantity $\left(t-s / c_{\varphi}\right)$ is positive.

\section{Centers of curvature of the wavefront}

Time-reversing the radiated wavefront results in a focal spot at the center of curvature of $\mathbf{F}^{ \pm}$. The radius of curvature $R_{c}$ of these curves is defined from the derivatives of its coordinates $x(t, \theta)$ and $y(t, \theta)$ with respect to $\theta$

$$
R_{c}(t, \theta)=\frac{\left(x^{\prime 2}+y^{\prime 2}\right)^{3 / 2}}{x^{\prime} y^{\prime \prime}-y^{\prime} x^{\prime \prime}},
$$

where' and " denote the derivatives with respect to $\theta$. The internal normal vector $\mathbf{N}_{\text {int }}$ of the curve is defined for each point $(x(t, \theta), y(t, \theta))$ by equation (10)

$$
\mathbf{N}_{\text {int }}(t, \theta)=\frac{1}{\sqrt{x^{\prime 2}+y^{\prime 2}}}\left\{\begin{array}{r}
-y^{\prime} \\
x^{\prime}
\end{array}\right.
$$

It is then possible to determine the centers of curvature $\mathbf{C}^{+}(t, \theta)$ and $\mathbf{C}^{-}(t, \theta)$ for each point of the wavefronts $\mathbf{F}^{ \pm}(t, \theta)$ following

$$
\mathbf{C}^{ \pm}(t, \theta)=\mathbf{F}^{ \pm}(t, \theta)+R_{c}(t, \theta) \mathbf{N}_{\text {int }}
$$

In the case of a circular shell of radius $\rho(\theta)=a$, we have $\gamma(\theta)=\theta$ and $s(0, \theta)=a \theta$. The above relations simplifies and lead to relations (12).

$$
\mathbf{C}_{\text {circle }}^{ \pm}(t, \theta)=\left\{\begin{aligned}
x_{c}(\theta) & =a \frac{c_{0}}{c_{\varphi}} \sin (\theta \pm \beta) \\
y_{c}(\theta) & =-a \frac{c_{0}}{c_{\varphi}} \cos (\theta \pm \beta)
\end{aligned}\right.
$$






Figure 6: Wavefronts around a circular shell for two circumferential waves (with $a=10 \mathrm{~mm}$ and $\left.c_{0} / c_{\varphi}=0.6\right)$. Solid line is for the counterclockwise guided wave and dashed line for the clockwise guided wave. The gray lines are the locus of the centers of curvature of the radiated wavefronts.

In this case, $\mathbf{C}_{\text {circle }}{ }^{ \pm}$does no longer depend on time, implying that it does not depend on the distance of observation. The centers of curvatures of the wavefronts are located on a circle of radius $a \frac{c_{0}}{c_{m}}$ centered on $O$. This result is consistent with those of Padilla [11] and can justify the equation (1) used in [12] and [13]. An example of radiated wavefronts and their associated centers of curvature is presented in figure 6 in the case of a circular shell. Due to the symmetry of the configuration, the set of centers of curvature are the same for both the counterclockwise and the clockwise wavefronts.

In the case of an elliptic shell, it is possible to obtain a semi-analytic result, involving incomplete elliptic integrals, but in the case of a shell of arbitrary cross section, the wavefronts and their centers of curvature (eqs. (7), (8) and (11)) have to be computed numerically. The case of an elliptic shell is represented in figure 7. The locus of the centers of curvature describe a rather complex shape compared to the case of a circular shell. Moreover the locus corresponding to each wavefront (clockwise and counterclockwise) are different. 




Figure 7: Wavefronts around an elliptic shell for two circumferential waves (with $r_{1}=10 \mathrm{~mm}$, $r_{2}=7.5 \mathrm{~mm}$ and $c_{0} / c_{\varphi}=0.6$ ). Plain line is for the counterclockwise guided wave and dashed line for the clockwise guided wave.

\section{OBSERVATION OF THE RADIATED WAVEFRONTS BY A TRANS- DUCER ARRAY}

In practice, only a small amount of the radiated wavefronts will reach an array of receivers, arising from a limited part of the shell. Accordingly, the time-reversed received signals will focus at a reduced number of centers of curvature. Considering the configuration presented in section II, figure 8 shows the centers of curvature of the part of the wavefronts reaching the array in the case of a circular shell (a) and of an elliptical shell (b, c). The points corresponding to the origin of the wavefronts are visible in light gray.

The centers of curvature $\mathbf{C}^{ \pm}$(in gray) are then reduced to a unique point, denoted $C_{0}$, which represents the centroid of the centers of curvature for a given wavefront, weighted by a Hamming window over the elements of the array (this apodization is chosen to attach greater importance to the central elements, leaving the external elements with a low but nonzero level). These $C_{0}$ points are represented with black points on figure 8 . We can notice that in the case of the elliptical shell the spatial distribution of the centers of curvature $\mathbf{C}^{ \pm}$ is limited (in the figure, the centers of curvature are often masked by the point representing their centroid $C_{0}$ ).

The expressions of $\mathbf{F}^{ \pm}(t, \theta)$ also depend on time. It is possible to evaluate the phase delay 


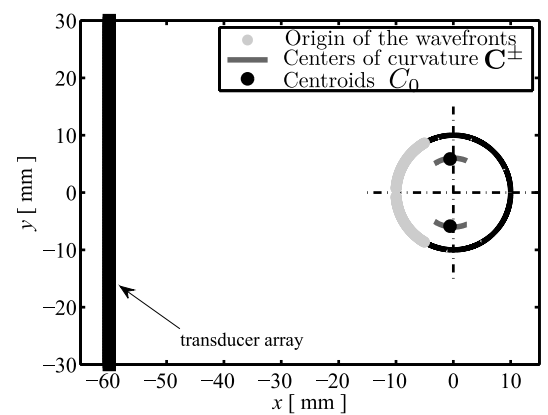

(a) Circular shell

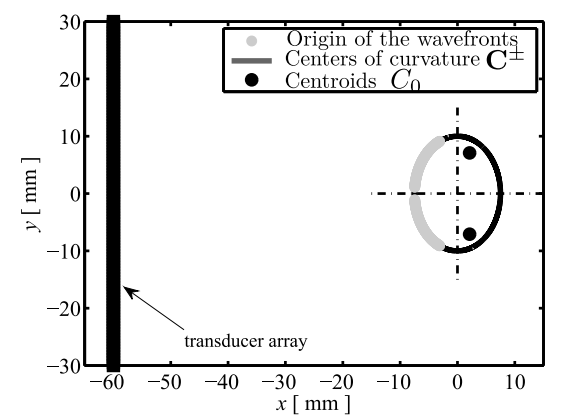

(b) Elliptic shell $\left(\alpha=0^{\circ}\right)$

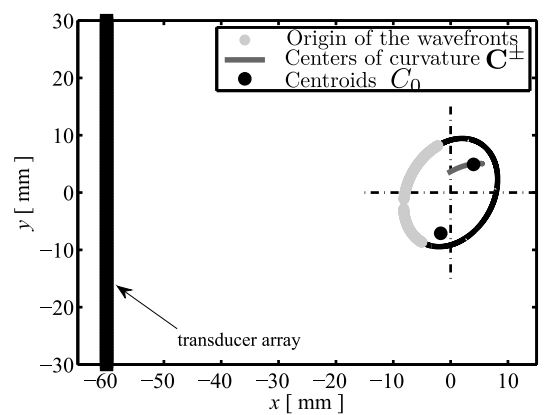

(C) Elliptic shell $\left(\alpha=30^{\circ}\right)$

Figure 8: Recording of the wavefronts by a transducer array for a circumferential wave, according to the configuration presented in section II and $c_{0} / c_{\varphi}=0.6$.

$\phi_{i}$ between the signals recorded for each element $i$ of the array.The time-reversal principle can then be applied using these delays: Assuming a frequency $f_{0}=1 \mathrm{MHz}$ and that each element acts as a point source in plain water with the wavenumber $k_{0}=\frac{2 \pi f_{0}}{c_{0}}$, the field $\Phi_{i}$ emitted by each element $i$ at the distance $r_{i}$ in free space is the classical two-dimensional Green's function in the far field approximation (eq. (13))

$$
\Phi_{i}\left(r_{i}, \phi_{i}\right)=\frac{\mathrm{e}^{i\left(k_{0} r_{i}+\phi_{i}\right)}}{\sqrt{r_{i}}} .
$$

Adding the fields $\Phi_{i}$ for each emitter of the array leads to a time-reversed focal spot. Some examples are presented on figure 9 for the 3 configurations of section II. The position of the maxima of the focal spots (white star) is very close to the centroids $C_{0}$ (white circles) of the centers of curvature $\mathbf{C}^{ \pm}$of the wavefronts. This confirms that the time reversal process focuses on the center of curvature of the wavefronts. This also shows that considering only the centroids $C_{0}$ instead of all the centers of curvature as in fig. 8 is sufficient to evaluate the position of the focal spots. For the sake of simplicity only the centroids $C_{0}$ will be considered in the following of the paper.

In the case of the circular shell, the two centroids $C_{0}$ corresponding to the two wavefronts in opposite directions are symmetric with respect to the horizontal $\vec{x}$ axis. Applying the time reversal process in this case lead to two focal spots close to the medium axis of the shell, that is at $x=0$, referred to as the $x_{0}$-axis in the following. Here, it is important to note that the method introduced in $[12,13]$ assumed that time-reversed signals of radiated circumferential waves focus on the $x_{0}$-axis, which is consistent with the results of figure 9 -a. On the contrary, 


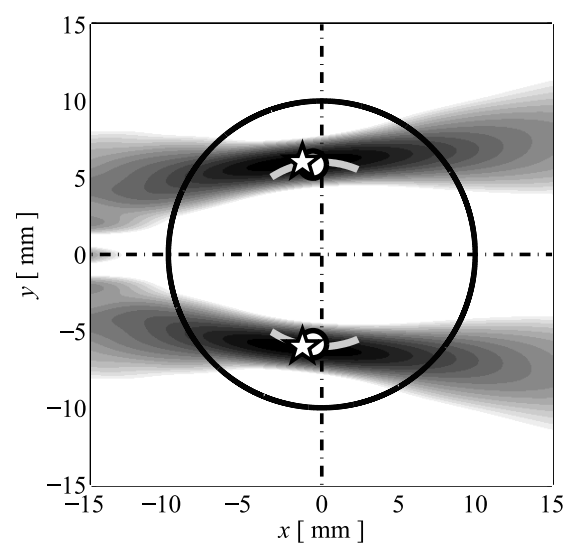

(a) Circular shell



(b) Elliptic shell $\left(\alpha=0^{\circ}\right)$

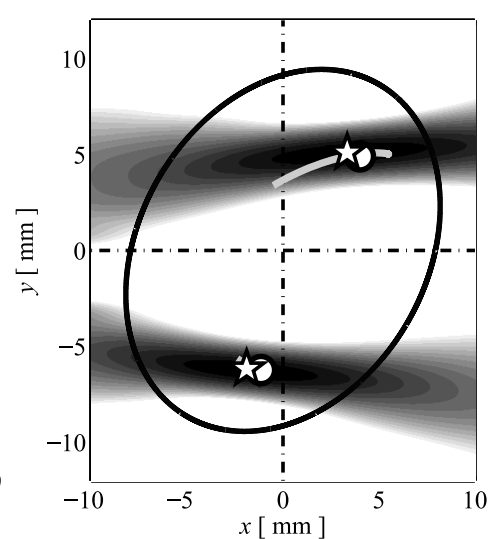

(C) Elliptic shell $\left(\alpha=30^{\circ}\right)$

Figure 9: Focal spots obtained by the back-propagation of the wavefronts for a circumferential wave with $c_{0} / c_{\varphi}=0.6$, at $f_{0}=1 \mathrm{MHz}$. The white stars indicates the maxima of the focused field, , the position of the $\mathbf{C}^{ \pm}$are presented in light gray line and the centroids $C_{0}$ are in white circles.

for an elliptic shell as in fig. 9-b the two focal spots are no longer on $x_{0}$. Moreover in a general case of fig. 9-c or with a shell of arbitrary cross section, the focal spots are also no longer symmetric with respect to the horizontal axis.

\section{PROPOSED METHOD TO DETERMINE CIRCUMFERENTIAL WAVES PHASE VELOCITIES}

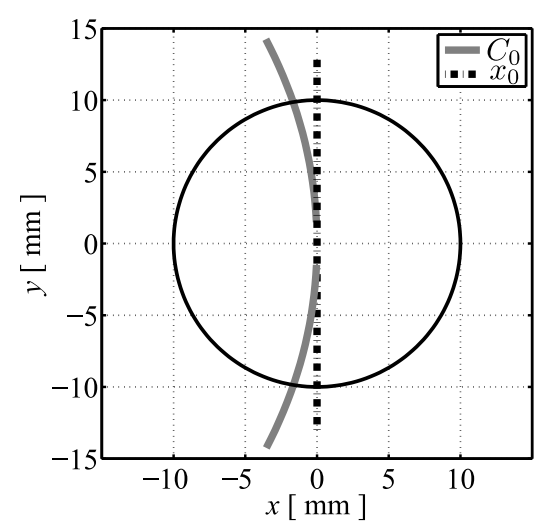

(a) Circular shell



(b) Elliptic shell $\left(\alpha=0^{\circ}\right)$



(C) Elliptic shell $\left(\alpha=30^{\circ}\right)$

Figure 10: $C_{0}$-curves for various shells with $c_{\varphi}$ varying from 1000 to $9000 \mathrm{~m} . \mathrm{s}^{-1}$. 


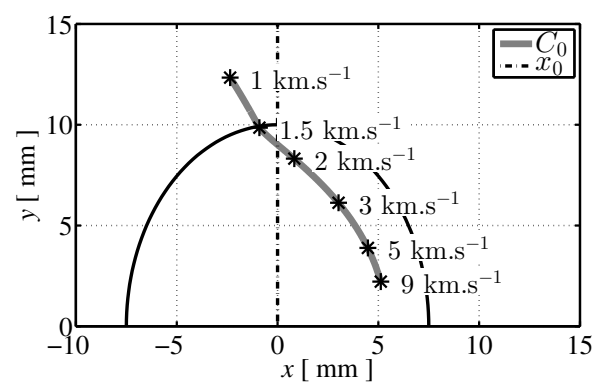

Figure 11: Details of the location of the $C_{0}$ points with their relative phase velocities $c_{\varphi}$ for the counterclockwise circumferential wave, in the case of an elliptic shell with major axis parallel to the transducer array.

The previous section has shown that a given phase velocity $c_{\varphi}$ of the circumferential wave leads to two particular positions of $C_{0}$ related to the counterclockwise and clockwise directions of the wave. Varying the phase velocity provides a set of spatial points $C_{0}$ which constitutes a parametric curve with parameter $c_{\varphi}$ referred to as the $C_{0^{-}}$-curve (fig. 10 ) in the following.

Figure 11 presents the details of the phase velocity values on a counterclockwise $C_{0}$-curve. The points of the curve located outside the shell correspond to $c_{\varphi}$ lower than the velocity $c_{0}$ in the surrounding fluid. This is similar to the case $d>D$ in eq. (1). Also, when $c_{0}=c_{\varphi}$ (here $1500 \mathrm{~m} \cdot \mathrm{s}^{-1}$ ) the point $C_{0}$ is located exactly on the surface of the shell. The part of the $C_{0}$-curve outside the shell can account for generalized Stoneley waves and Franz waves (creeping waves).

Figure 11 shows that the points of the $C_{0}$-curve towards the center of the object, corresponding to the highest values of $c_{\varphi}$, are closer one to the others than the points corresponding to the lowest values of $c_{\varphi}$. In other words, the distribution of $c_{\varphi}$ along the curve is denser as the velocity increases. This is illustrated, for example, by the 'short' distance between the points corresponding to $c_{\varphi}=5$ and $9 \mathrm{~km} \cdot \mathrm{s}^{-1}$, compared to the relatively 'long' distance between $c_{\varphi}=2$ and $3 \mathrm{~km} . \mathrm{s}^{-1}$.

The principle of the proposed method to measure phase velocities of circumferential waves is to use a predefined $C_{0}$-curve. Such a curve can be calculated knowing only the position and external geometrical shape of the shell. Note that there is no hypothesis made over the 
thickness of the shell nor on the material properties: only the propagation of a circumferential wave with a given phase velocity is assumed. The proposed method is outlined below:

1. Calculate with eq. (11) the $C_{0}$-curve corresponding to the measured object (position, external geometry and range of expected phase velocities), given the relative positions and size of the object and the transducer ;

2. Excite circumferential waves in the shell and record the waves radiated in the surrounding medium with a transducer array;

3. Fourier-transform the recorded signals;

4. In cases where time-domain signals of several circumferential modes overlap, the DORT method can be used to separate the contribution of each mode and eliminate the non significant contributions along with noise;

5. Calculate, for each frequency, the amplitude of the back-propagated wavefield along the predefined $C_{0}$-curve. The phase velocity of the circumferential wave (or of the mode selected at step $\sharp 4$ in cases where several guided modes exist) corresponds to the point of the $C_{0}$-curve where the wavefield is maximum, that is, in practice, at the intersection between the $C_{0}$-curve and a focal spot.

The method involving the use of eq. (1) and (2) can also be used but only in the case (a) and (b) of figure 10, where the shell is symmetric relative to the $x$-axis. In this case it is sufficient to calculate the back-propagated wavefield on the $x_{0}$-axis, measure the distance $d$ between the two focal spots and apply the analytic equations to recover the phase velocities. This much simpler method will be referred as the " $x_{0}$ method" in the following. In the case of a circular shell (fig 10-a), the $C_{0}$ curve is very close to the medium axis of the shell $x_{0}$. In this case the use of the $x_{0}$ method may be sufficient to compute the dispersion curve. In the case of the elliptic shell with a major axis parallel to the transducer array (fig 10-b), as the $C_{0}$ curve is rather different from the $x_{0}$ curve, the $x_{0}$ method may still be practicable but will provide different results. In the general case of shell with arbitrary cross section, the $x_{0}$ method is not practicable. 
The heterogeneous distribution of $c_{\varphi}$ values along the $C_{0}$-curve discussed above has important consequences as a better resolution will be obtained for the estimations of low $c_{\varphi}$ than of high $c_{\varphi}$. In particular in the non symmetric case (here in fig 10-c) the $C_{0}$ curve in the lower part corresponding to the centroids for the clockwise circumferential wave is quite short and all points are close one to another; it will be more difficult to distinguish different phase velocities as the focal spots will cover a large amount of these points. On the contrary the upper part presents points spreaded in space and then provide a better resolution on $c_{\varphi}$.

\section{NUMERICAL VALIDATION}

Numerical simulations have been used to validate our approach. Simulations were performed with a two-dimensional Time-Domain Finite Difference (FDTD) code SimSonic [21, 24]. The configuration of the simulations is the same as described in section II.

In order to perform the Singular Value Decomposition (SVD) needed by the DORT method [14, 13, 25], the response function of the transducer array was obtained with emissions from 32 elements evenly spaced along the array. The reception is performed with all the 128 elements. The source signal emitted by each element of the array is a Ricker wavelet (or Mexican hat) with a central frequency $f_{0}=1 \mathrm{MHz}$. The spatial step of the simulation grid is $h=0.025 \mathrm{~mm}$ and a preliminary study on homogeneous mediums has shown the numerical dispersion to be much less than $1 \%$ in that case.

The dispersion curves of guided waves modes measured with the proposed method are compared to reference curves calculated for a plane plate loaded with water on one side and free on the other side [26].

Figure 12 presents an example of snapshot recorded during the simulations. The presence of several modes of circumferential waves propagating around the shell is visible, along with the wavefronts radiated in water. The shape of these simulated wavefronts is similar to the geometrical shapes computed with equations (7) and (8).

As there are several modes of propagation for the circumferential waves, each of them radiating in the fluid, several radiated wavefronts interfere. The use of the DORT method on the recorded signals at the receivers will allow to separate the major contributions of the radiated field. This is done by considering only a few high singular vectors of the time 


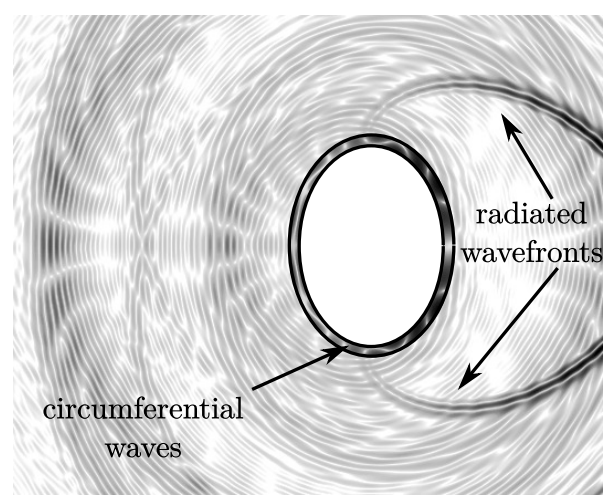

Figure 12: Snapshot of the simulation of circumferential waves around an elliptic shell and radiating in the surrounding medium.

reversal operator, discarding the others. Doing so eliminates all the components with a low signal-to-noise ratio. The highest singular vectors are used to calculate the retro-propagated wavefield on the $C_{0}$-curve calculated as indicated in the previous sections. When possible (case of a circular shell or an elliptic shell with major axis parallel to the array), the $x_{0}$ method will also be performed as a way of comparison. As these two cases are symmetric with respect to the horizontal axis, only the counterclockwise wave i.e. the upper part of the shell will be considered.

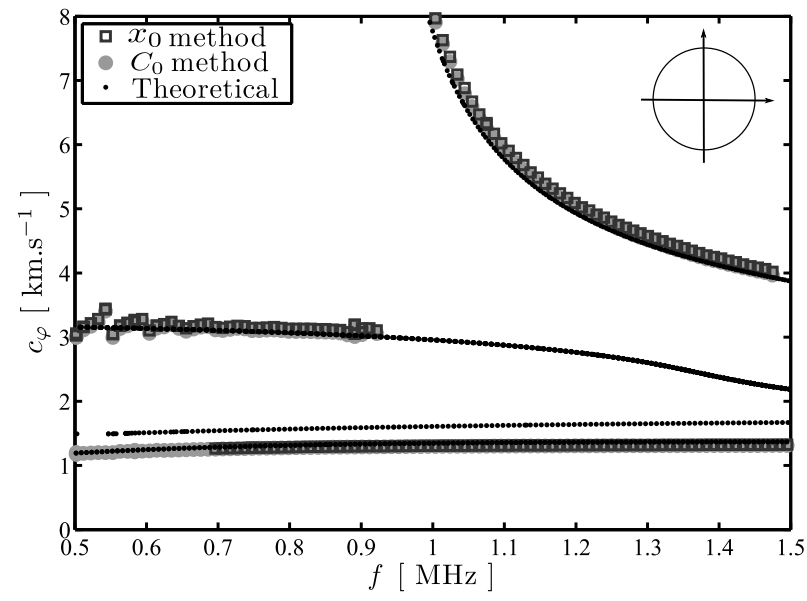

Figure 13: Dispersion curves for a circular shell of thickness $e=1 \mathrm{~mm}$. The squares represents the results obtained from the $x_{0}$ method (eq. (1)), and the gray rounds represents the results obtained with the $C_{0}$ method.

The results obtained on a circular shell are presented on figure 13. The classical $x_{0}$ method 
and the $C_{0}$ method provide almost the same results, as expected from fig. 10-a where the $C_{0}$-curve is very close to the $x_{0}$-axis. The agreement with the theoretical dispersion curves is very good and allows to recover the branches identified as the S0 and the A1 Lamb modes. The lower branch with a velocity close to $1000 \mathrm{~m} . \mathrm{s}^{-1}$ corresponds to the generalized Stoneley wave at the interface with the shell. These excellent results on the circular shell validate our approach and approximations, along with the simulations tools used. In the following, only the external shape of the shell is changed.

Figure 14 presents the results for an elliptic shell with its major axis parallel to the transducer array. Significant discrepancies are found between the results of the $x_{0^{-}}$and $C_{0^{-}}$ methods, especially for the higher velocities (A1 mode). The results from the $C_{0}$ method are closer to the reference theoretical curves than those of the $x_{0}$ method. They are within $10 \%$ of the reference values.

For an elliptic shell with an arbitrary inclination the $x_{0}$ method is not available (Fig. 15). Due to the non-symmetry of the shell related to the horizontal axis, the branches of the dispersion curves may be evaluated considering the counterclockwise circumferential waves (upper part of the shell) or the clockwise waves (lower part), leading to different results. Using counterclockwise waves, the agreement with the theoretical modes is excellent while it is less good using clockwise waves This may be partly explained considering the positions of the $C_{0}$ points on fig. 10-c, where the points corresponding to the upper part are more spreaded in space leading to a better resolution of the method.

\section{DISCUSSION AND CONCLUSION}

The paper presented a method to estimate the phase velocity of the circumferential waves around thin shells in a non contact way. It applies to shells of any shape, provided the external geometry is known, along with the position and size of the transducer array. As the computation of the $C_{0}$ curves only depend on the external geometry of the shell, this method may also be suitable to study shells with varying thickness. In the same way, the mechanical properties of the shell may vary along the shell. In these cases the estimation of the phase velocities is only related to the part of the shell where the wavefronts recorded by the receivers were emitted. The computation of $\mathbf{F}^{ \pm}(t, \theta)$ allows determining the origins 




Figure 14: Dispersion curves for an elliptic shell $(e=1 \mathrm{~mm})$ which major axis parallel to the transducer array. The squares represents the results obtained from the $x_{0}$ method (eq. (2)), and the gray circles represents the results obtained with the $C_{0}$ method.

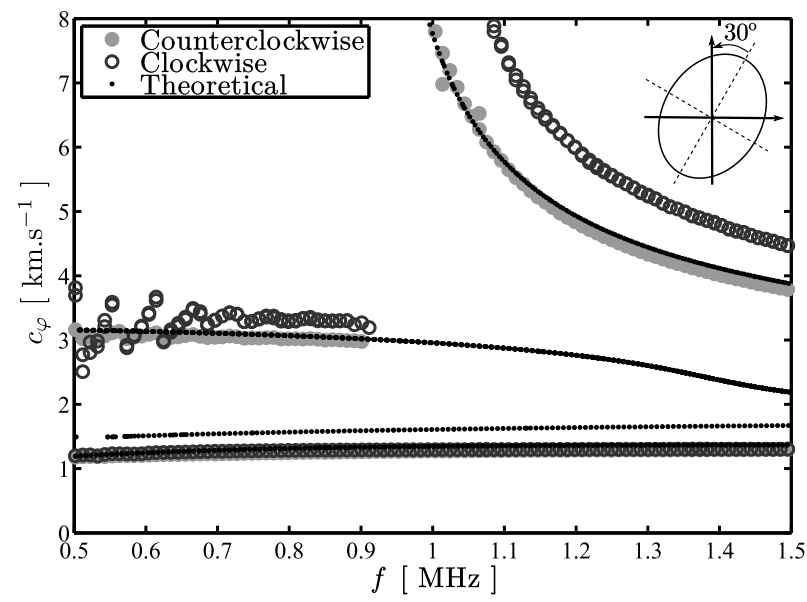

Figure 15: Dispersion curves for an elliptic shell $(e=1 \mathrm{~mm})$ with an inclination of $30^{\circ}$.

of the wavefronts reaching the receivers (see for example fig. 8) and thus the portion of the shell investigated.

However there are a few limitations to the technique. First the circumferential waves have to be generated in the shell by the incident wave from the transducer. Some modes of circumferential may not be excited and thus their associated branches in the dispersion curve will not be estimated. Second, the circumferential waves also have to propagate around the shell and radiate in the surrounding medium. This may induce an important attenuation: if the attenuation is too important the circumferential wave will not be able to circle around 
the shell and to radiate toward the receiver.

When considering a geometry of shell, it is important to look at the shape of the $C_{0}$-curve, and in particular at the distribution of the velocities along this curve. In practice, if the $C_{0}$ points corresponding to different phase velocities are too close, the focal spots of the time reversal process will not be able to distinguish between them. Therefore, the orientation of the shell relative to the position of the array is important : an orientation which leads to the $C_{0}$ points the more spreaded in space as possible is preferred. In any cases, the resolution for lower velocities is much better than for the velocities greater than $3-4 \mathrm{~km} . \mathrm{s}^{-1}$. Another limitation is that all the dimensions and the relative positions have to be known precisely, as a small error in the position of the $C_{0}$-curve $(<1 \mathrm{~mm})$ may lead to a difference greater than $500 \mathrm{~m} \cdot \mathrm{s}^{-1}$.

For the non-circular cases, e.g. fig 14 and 15, the maximal error with the reference value is about $10 \%$ for high velocities. As the results obtained with the circular shell are excellent, this error cannot come from the simulation tool used, nor from the fact that the curvature of the shell is neglected in the reference value (Lamb waves in a plane plate). The origin of the error is still not fully explained and has to be investigated further.

The method presented here can be useful in medical applications, for example to evaluate the mechanical properties of bones like femoral neck in vitro or in vivo where the cortical (compact) part of the bone can be considered as a shell of varying geometry, thickness and properties, immersed in a fluid. Some industrial application may also be found as the method is suitable for a non contact inspection and evaluation of any tubular component, like pipes for instance.

\section{ACKNOWLEDGEMENTS}

The authors wish to thank Dr Maryline Talmant and Dr Pierre Nauleau for her helpful comments and discussions. The authors are grateful for the financial support of UPMC in the framework of the program Emergence 2009 (Post-Doctoral Fellowship M. Chekroun). 


\section{References}

[1] R. D. Doolittle, H. Uberall, and P. Ugincius. Sound scattering by elastic cylinders. J. Acoust. Soc. Am., 43(1):1-14, 1968.

[2] G. V. Frisk, J.W. Dickey, and H. Uberall. Surface wave modes on elastic cylinders. J. Acoust. Soc. Am., 58(5):996-1008, 1975.

[3] G. V. Frisk and H. Uberall. Creeping waves and lateral waves in acoustic scattering by large elastic cylinders. J. Acoust. Soc. Am., 59(1):46-54, 1976.

[4] J.B. Keller. Geometrical theory of diffraction. J. Opt. Soc. Am., 52(2):116-130, 1962.

[5] Philip L. Marston. GTD for backscattering from elastic spheres and cylinders in water and the coupling of surface elastic waves with the acoustic field. J. Acoust. Soc. Am., $83(1): 25-37,1988$.

[6] W. G. Neubauer. Pulsed Circumferential Waves on Aluminum Cylinders in Water. J. Acoust. Soc. Am., 45(5):1134-1144, 1969.

[7] N. Gespa. La diffusion acoustique par des cibles élastiques de forme géométrique simple: Theorie et Experiences. Centre de Documentation de l'Armement, Paris, 639 pages, 1987.

[8] Z. Wu, G. Zhou, and S. Feng. Visalization of the sound scattering by a cylinder. Acta Acustica, 18:81-90, 1993.

[9] E. Heyman and L. B. Felsen. Traveling wave and SEM representations for transient scattering by a circular cylinder. J. Acoust. Soc. Am., 79(2):230-238, 1986.

[10] W. Hassan and P. B. Nagy. Circumferential creeping waves around a fluid-filled cylindrical cavity in an elastic medium. J. Acoust. Soc. Am., 101(5):2496-2503, 1997.

[11] F. Padilla, B. Poirée, M. Talmant, and G. Quentin. The wave bivector formalism associated with circumferential leaky waves. J. Acoust. Soc. Am., 108(1):25-30, 2000. 
[12] J.L. Thomas, P. Roux, and M. Fink. Inverse Scattering with an Acoustic Time-Reversal Mirror. Phys. Rev. Lett., 72:637-640, 1994.

[13] C. Prada and M. Fink. Separation of interfering acoustic scattered signals using the invariants of the time-reversal operator. Application to Lamb waves characterisation. $J$. Acoust. Soc. Am., 104(2):801-807, 1998.

[14] C. Prada and M. Fink. Eigenmodes of the time reversal operator: A solution to selective focusing in multiple-target media. Wave Motion, 20(2):151 - 163, 1994.

[15] P. Nauleau, E. Cochard, J.-G. Minonzio, Q. Grimal, P. Laugier, and C. Prada. Characterization of circumferential guided waves in a cylindrical cortical bone-mimicking phantom. J. Acoust. Soc. Am., 131(4):EL289-EL294, 2012.

[16] P. Nauleau, Q. Grimal, J.-G. Minonzio, P. Laugier, and C. Prada. Circumferential guided wave measurements of a cylindrical fluid-filled bone-mimicking phantom. $J$. Acoust. Soc. Am., 135(2):994-1001, 2014.

[17] M. Chekroun, Q. Grimal, E Cochard, J.-G. Minonzio, C. Prada, and P Laugier. Mesure des courbes de dispersion des ondes guidées circonférentielles dans une coque elliptique par retournement temporel. (Measurement of circumferential guided waves dispersion curves in an elliptic shell using time reversal). In proceedings of the $20^{\text {th }}$ Congrés Français de Mécanique, Besançon, France, august 2011. http://hdl .handle.net/2042/46432 (date last viewed $3 / 23 / 15$ ).

[18] G.S. Sammelmann, D. H. Trivett, and R. H. Hackman. The acoustic scattering by a submerged, spherical shell. I: The bifurcation of the dispersion curve for the spherical antisymmetric Lamb wave. J. Acoust. Soc. Am., 85(1):114-124, 1989.

[19] M. Talmant, J. M. Conoir, and J.-L. Rousselot. High frequency scattering by shells. Acta Acustica, 3:509-515, 1995.

[20] G. Maze, F. Leon, J. Ripoche, and H. Uberall. Repulsion phenomena in the phasevelocity dispersion curves of circumferential waves on elastic cylindrical shells. J. Acoust. Soc. Am., 105(3):1695-1701, 1999. 
[21] E. Bossy, M. Talmant, and P. Laugier. Three-dimensional simulations of ultrasonic axial transmission velocity measurement on cortical bone models. J. Acoust. Soc. Am., $115: 2314-2324,2004$.

[22] K. L. Williams and P. L. Marston. Backscattering from an elastic sphere: SommerfeldWatson transformation and experimental confirmation. J. Acoust. Soc. Am., 78(3):1093-1102, 1985.

[23] M. Fink. Time-Reversal of ultrasonic fields-Part I : Basic principles. IEEE T. Ultrason. Ferr., 39(5):555-566, 1992.

[24] Emmanuel Bossy. Simsonic software suite for the simulation of ultrasound propagation. http://www.simsonic.fr/ (date last viewed 3/23/15), 2012.

[25] J.-G. Minonzio, C. Prada, D. Chambers, D. Clorennec, and M. Fink. Characterization of subwavelength elastic cylinders with the decomposition of the time-reversal operator: Theory and experiment. J. Acoust. Soc. Am., 117(2):789-798, 2005.

[26] B Pavlakovic and M Lowe. Disperse software, v. 2.0. 16. Mechanical Engineering, Imperial College, London, 2005. 


\section{List of Figures}

1 Geometrical construction of the radiated wavefront by a circumferential wave around a circular cylinder (adapted from ref. [6]) . . . . . . . . . . . . . 3

2 General configuration of the setup used to evaluate the dispersion curves of the circumferential guided waves around a thin cylindrical shell. . . . . . . . 5

3 Principle of the generation and radiation of circumferential waves around a thin circular cylinder. . . . . . . . . . . . . . . . . . 6

4 Notations used to describe the radiation of a circumferential wave around a shell of arbitrary cross section. . . . . . . . . . . . . . . . . . 7

5 Direction of the radiation $\vec{k}^{+}$and $\vec{k}^{-}$for a circumferential wave propagating counterclockwise and clockwise. . . . . . . . . . . . . . . 8

6 Wavefronts around a circular shell for two circumferential waves (with $a=10 \mathrm{~mm}$ and $\left.c_{0} / c_{\varphi}=0.6\right)$. Solid line is for the counterclockwise guided wave and dashed line for the clockwise guided wave. The gray lines are the locus of the centers of curvature of the radiated wavefronts. . . . . . . . . . . . . . . 10

7 Wavefronts around an elliptic shell for two circumferential waves (with $r_{1}=10 \mathrm{~mm}$, $r_{2}=7.5 \mathrm{~mm}$ and $\left.c_{0} / c_{\varphi}=0.6\right)$. Plain line is for the counterclockwise guided wave and dashed line for the clockwise guided wave. . . . . . . . . . . . 11

8 Recording of the wavefronts by a transducer array for a circumferential wave, according to the configuration presented in section II and $c_{0} / c_{\varphi}=0.6$. . . . 12

9 Focal spots obtained by the back-propagation of the wavefronts for a circumferential wave with $c_{0} / c_{\varphi}=0.6$, at $f_{0}=1 \mathrm{MHz}$. The white stars indicates the maxima of the focused field, , the position of the $\mathbf{C}^{ \pm}$are presented in light gray line and the centroids $C_{0}$ are in white circles. . . . . . . . . . . 13

$10 C_{0}$-curves for various shells with $c_{\varphi}$ varying from 1000 to $9000 \mathrm{~m} . \mathrm{s}^{-1} \ldots \ldots$

11 Details of the location of the $C_{0}$ points with their relative phase velocities $c_{\varphi}$ for the counterclockwise circumferential wave, in the case of an elliptic shell with major axis parallel to the transducer array. . . . . . . . . . . . . . 14 
12 Snapshot of the simulation of circumferential waves around an elliptic shell and radiating in the surrounding medium. . . . . . . . . . . . . 17

13 Dispersion curves for a circular shell of thickness $e=1 \mathrm{~mm}$. The squares represents the results obtained from the $x_{0}$ method (eq. (1)), and the gray rounds represents the results obtained with the $C_{0}$ method. . . . . . . . . . .

14 Dispersion curves for an elliptic shell $(e=1 \mathrm{~mm})$ which major axis parallel to the transducer array. The squares represents the results obtained from the $x_{0}$ method (eq. (2)), and the gray circles represents the results obtained with the

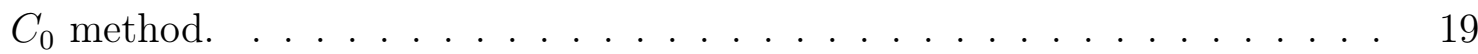

15 Dispersion curves for an elliptic shell $(e=1 \mathrm{~mm})$ with an inclination of $30^{\circ} . \quad 19$ 